\title{
Performance Evaluation of Coordinated Multi-Point Transmission and Reception in Indoor Mobile Communication Systems
}

\author{
Woongsup Lee ${ }^{1}$ and Howon Lee ${ }^{2 *}$, Member, KIICE \\ ${ }^{1}$ Institute for Digital Communications, University Erlangen-Nürnberg, Erlangen, D-91058, Germany \\ ${ }^{2}$ Department of Electrical, Electronic and Control Engineering \& IITC, Hankyong National University, Anseong 456-749, Korea
}

\begin{abstract}
Recently, mobile communication systems are suffering from exponentially increasing data traffic. As a promising solution to the increase in data traffic, a coordinated multi-point transmission and reception (CoMP) scheme has been proposed. Although a great deal of research has been done on this new technology, the performance of mobile communication systems with CoMP has not been evaluated properly in a typical indoor environment. To address this, we have developed a system-level simulator and evaluated the performance of mobile communication systems with CoMP. Unlike previous works, we have used an actual antenna pattern in our simulator and link-level results are properly taken into account through link-level abstraction. By using a system-level simulator, we have evaluated the performance of mobile communication systems with CoMP in an indoor environment and found that unlike an outdoor cellular environment, CoMP may not improve the performance of overall mobile communication systems in an indoor environment.
\end{abstract}

Index Terms: CoMP, Indoor mobile communication systems, Performance evaluation, System-level simulator

\section{INTRODUCTION}

Ever since Guglielmo Marconi succeeded in transmitting data over a wireless channel, the impact of wireless communication technology on our daily lives has been increasing due to its convenience. As wireless communication technology is utilized more extensively, it becomes more difficult to cope with high traffic demands since the spectrum for wireless communication is limited. In recent days, mobile data traffic, in particular, has been increasing exponentially [1]. In fact, the total amount of mobile data traffic in 2013 is twelve times larger than all internet traffic in 2000 [1].

To cope with this ever increasing traffic demand, a number of new wireless technologies have been considered. For example, orthogonal frequency division multiplexing (OFDM) has played a significant role in improving the capacity of third generation mobile communication systems [2]. In addition to OFDM, multiple input multiple output (MIMO) and femtocells have also been studied extensively as the next key technologies for mobile communication systems in order to improve their capacity [3, 4]. Moreover, new concepts in wireless communication systems have been considered, such as cognitive radio, in which, without having licenses to use bands, wireless systems opportunistically utilize bands when the bands are empty [5]. Furthermore, highly directive beam-forming techniques have been considered [6].

Received 05 April 2013, Revised 06 May 2013, Accepted 04 June 2013

*Corresponding Author Howon Lee (E-mail: hwlee@hknu.ac.kr, Tel: +82-31-670-5198)

Department of Electrical, Electronic and Control Engineering \& IITC, Hankyong National University, 327 Jungang-ro, Anseong 456-749, Korea.

(c) This is an Open Access article distributed under the terms of the Creative Commons Attribution Non-Commercial License (http://creativecommons.org/li-censes/by$\mathrm{nc} / 3.0 /$ ) which permits unrestricted non-commercial use, distribution, and reproduction in any medium, provided the original work is properly cited. 
One of the promising technologies to improve the capacity of the mobile communications system is coordinated multi-point transmission and reception (CoMP) [710]. In CoMP, the interference between neighboring base stations (BSs) is controlled efficiently, such that the users in the cell edge can improve the signal-to-interference noise ratio (SINR), that is, they can improve the data rate. Given that inter-cell interference is the one dominant factor in the deterioration of the performance of the cell-edge users, the performance of mobile communication systems can be greatly improved by utilizing CoMP technology. CoMP is one of the dominant technologies in the 3GPP long-term evolution advanced (LTE-A) standard to improve the performance of mobile communication systems.

Although the performance of mobile communication systems with CoMP has been extensively evaluated in previous works [7-10], the performance evaluations have been conducted in outdoor cellular systems. However, it is expected that $70 \%$ of total data traffic will be generated in the indoor environment; thus, it would be beneficial to evaluate the performance of CoMP in the indoor environment [1]. Moreover, previous performance evaluations have not properly taken into account the actual radio environment, such as the actual radiation pattern of the antennae in an indoor BS. Furthermore, for most performance evaluations based on system-level simulators, the link level of the communication protocol has been highly simplified. To solve these problems with current approaches, we have developed a system-level simulator by considering the actual indoor radio environment and evaluated the performance of indoor mobile communication systems with CoMP in realistic environments. The key finding of our results is that CoMP may not improve the performance of indoor mobile communication systems due to strong interference from other neighboring BSs that do not cooperate in CoMP. To fully take advantage of the benefits of CoMP in the indoor environment, the number of BSs that cooperate in CoMP should be increased compared to outdoor cellular systems.

The remainder of this paper is organized as follows: In Section II, we introduce CoMP technology proposed to increase the capacity of mobile communication systems, and explain several aspects in our indoor system level simulator in Section III. In Section IV, we evaluate the performance of mobile communication systems with CoMP. In Section V, we draw conclusions.

\section{COMP OVERVIEW}

In this chapter, we will briefly explain the concept of CoMP and how CoMP operates in our system-level simulator. We will also explain the details of our system- level simulator and highlight the major differences between our work and previous works.

The essence of CoMP is that neighboring BSs cooperate in transmission such that designated users can obtain a better data rate. For this, there are two major schemes in CoMP: coordinate scheduling/coordinate beamforming (CS/ $\mathrm{CB}$ ) and joint processing (JP). In CS/CB, two neighboring BSs transmit data to two distinct users such that the effect of each transmission of data on other data transmission can be minimized. More specifically, each BS schedules users such that the interference between two data transmissions can be minimized (CS). Moreover, each transmission is controlled such that interference can be minimized (CB). In CB, specially designed beamforming can be utilized. By using $\mathrm{CS} / \mathrm{CB}$, two distinct data flows can be conveyed while the interference between these two data flows is reduced to improve the quality of data transmission. Fig. 1(a) provides more details.

On the other hand, in JP, two BSs transmit the same data to one common user or only one BS transmits the data to a user. The former scheme is referred as joint transmission (JT), and the latter scheme is referred to as dynamic cell selection. Fig. 1(b) provides more details. Unlike CS/CB, in JP only one data flow will be conveyed and the data transmission opportunity has been reduced by half. However, dominant interference will be completely removed (dynamic cell selection) or transformed into a designated signal (JT) such that the improvement in the SINR of CoMP users will be much greater than in the case of the CS/CB. With regard to improving a cell-edge user's data rate, JP is more effective than CS/CB. Therefore, in this paper, we will mainly focus on the JT scheme.

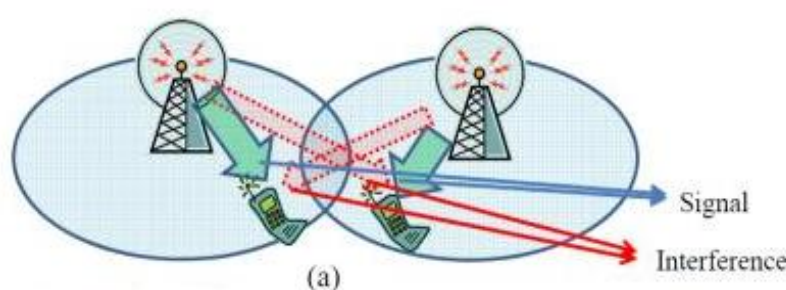

(a)

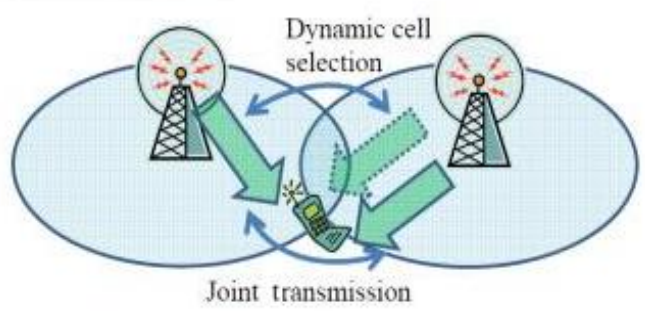

(b)

Fig. 1. Coordinated multi-point transmission and reception overview: (a) coordinated scheduling/coordinated beamforming and (b) joint processing. 
In our system level simulator, a user will receive data by using CoMP, which is based on JT, if the ratio of the interference power from a specific neighboring BS and the signal power from its associated BS is larger than CoMP_ratio. Therefore, CoMP_ratio is the minimum required ratio of the dominant interference and signal for CoMP to be used, and the set of users that use CoMP can be controlled by adjusting CoMP_ratio. We have made such a selection criterion because CoMP will work well only when the dominant interference is sufficiently large. Moreover, the portion of time that is assigned for CoMP is proportional to the number of users that use the CoMP. It should be noted that one transmission opportunity will be lost by using CoMP.

\section{SEVERAL ASPECTS OF THE INDOOR SYSTEM-LEVEL SIMULATOR}

Herein, we will explain the key characteristics of our system-level simulator and the simulation environments that we have implemented in our system-level simulator.

\section{A. Antenna Pattern}

In our system-level simulator, we have used a realistic antenna pattern, which is obtained from real measurements. We have integrated two antennas into one antenna unit. The two antennas in the antenna unit are placed at +45 and -45 degrees, such that these two antennas have different polarization. In this implementation, cross correlation between the two antennas can be maintained at less than $-30 \mathrm{~dB}$. We have placed two antenna units in each BS and user. Therefore, each BS and user will have four antennas in total. Fig. 1 shows the measured antenna pattern for our antenna configuration. The figure, shows that the directional antenna gain is about $8 \mathrm{dBi}$. We have used this antenna pattern in our system level simulator. By utilizing a realistic antenna pattern, the system level simulator will generate simulation results with higher accuracy.

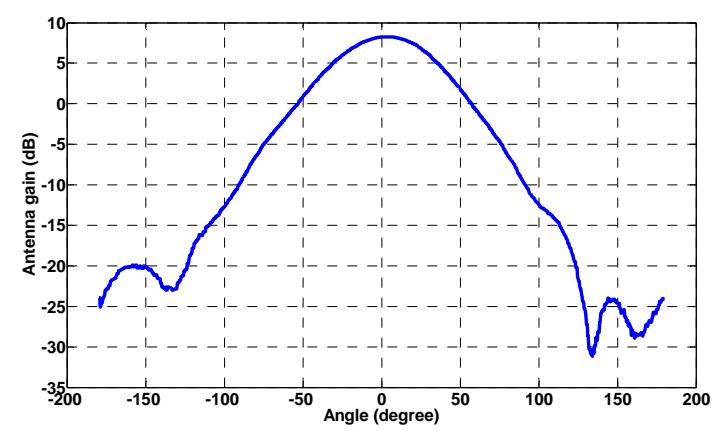

Fig. 2. The antenna pattern used in our system level simulator.

\section{B. Link-Level Abstraction}

In our system-level simulator, we have used link-level abstraction to emulate the link-level operations of communication links $[6,11]$. For this, we have used the LTE linklevel simulator and evaluated the packet error rate (PER) for all the possible combinations of SINR and modulation and coding scheme levels. We have also summarized all the results on the PER. In the system-level simulator, we have only calculated the SINR for the communication links and found the corresponding PER value for the SINR from the simulation results from the link-level simulator. After that, we have dropped the packets with the same probability as the PER value. This kind of link-level abstraction has been widely used in previous works in implementing systemlevel simulators $[6,11]$, and the link-level of communication links can be properly taken into account by using this approach.

\section{Other Simulation Environment}

We have designed a system-level simulator for the indoor environment. The basic simulation environment is the same as the indoor hotspot scenario in ITU-R M.2135 [12]. A specific band for 3GPP LTE service in South Korea has been taken into account, that is, downlink (1,840-1,850 MHz) and uplink (1,745-1,755 MHz). Each BS is configured to satisfy the 3GPP TS 36.104 ACLR $46 \mathrm{dBc}$ standard [13] and the transmission power of the BS is set to $1 \mathrm{~W}$. In our systemlevel simulator, we assume that the antenna configuration is 4-by-4, and the data is transmitted by using multiuser-MIMO. We assume that four BSs are deployed inside of the building. By referring to the indoor hotspot scenario, we assume that the shape of the building is square and there are nine square rooms in the building, where the size of each room is $225 \mathrm{~m}^{2}$. As we can see from the antenna pattern in Fig. 2, the antenna that we have considered in our simulator has highly directive transmission characteristics. To properly give mobile service to all users in the area inside the building, we have placed four BSs at the corner. Moreover, users are placed within the building randomly according to a Poisson point process [14].

For scheduling, we assume that users who do not use CoMP have been scheduled by using proportional fair scheduling. Other users who use CoMP have been scheduled by using round-robin scheduling. We used roundrobin scheduling for CoMP users because 1) two BSs transmit data to one common user in CoMP and it is very complicated to calculate the PF index and 2) round-robin scheduling is fairer than proportional fair scheduling. Given that users who use the CoMP will be likely to be in bad channel conditions, it will be better to use fairer scheduling. Other simulation parameters are shown in Table 1. 
Table 1. Simulation parameters

\begin{tabular}{lc}
\hline \multicolumn{1}{c}{ Parameter } & Value \\
\hline RF carrier frequency (GHz) & 1.85 \\
Channel bandwidth (MHz) & 10 \\
Downlink subchannel & PDSCH \\
Antenna configuration & 4 -by-4 \\
Multipath fading model & Rayleigh channel model \\
BS transmission power (dBm) & 30 \\
Noise power (dBm) & -174 \\
Number of users in one cell & 10 \\
Path-loss model [12] & $43.3 * \log _{10}(\mathrm{~d})+16.9434$ \\
Wall loss (dBm) & 13 \\
Target PER (\%) & 1 \\
Scheduling & Non-comp user \\
$\quad$ Proportional fair scheduling & Comp user \\
Round-robin scheduling &
\end{tabular}

RF: radio-frequency, PDSCH: physical downlink shared channel, PER: packet error rate.

\section{SIMULATION RESULTS}

In this section, we will show the performance of indoor mobile communication systems with CoMP, which is based on the 3GPP LTE standard.

First, we checked the distribution of users who use CoMP by changing the CoMP_ratio parameter. The distribution of the users is shown in Figs. 3 and 4. In these figures, four BSs are located at the four corners of the building. As can be seen from the results, users located at the boundary of the cell are likely to use CoMP. This behavior coincides with our investigation.

Moreover, we can observe that more users are using the CoMP as the CoMP_ratio decreases. In the CoMP scheme that we have considered in our system-level simulator, a user is eligible to use CoMP only if the ratio of its designated signal power and the dominant interference power is larger than CoMP_ratio. Therefore, as CoMP_ ratio becomes smaller, more users can use CoMP, and the ratio of users that use CoMP increases.

Furthermore, we found that users located at the center of the building are more likely to use CoMP because the amount of interference becomes larger. In the room located at the center of the building, the designated signal from an associated BS and interference from the other three BSs should pass through the same number of walls (2 walls) and path loss is likely to be similar for the signal and the interference, which results in a higher probability for the CoMP criterion to be satisfied.

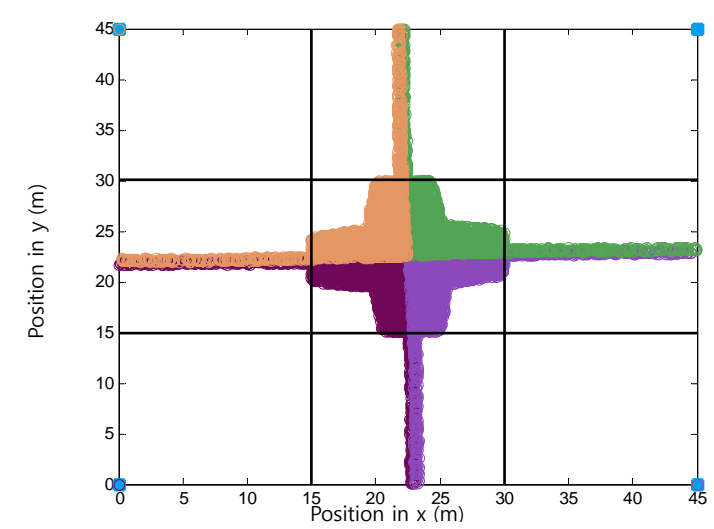

Fig. 3. Distribution of coordinated multi-point transmission and reception (CoMP) users when CoMP_ratio $=0.5$.

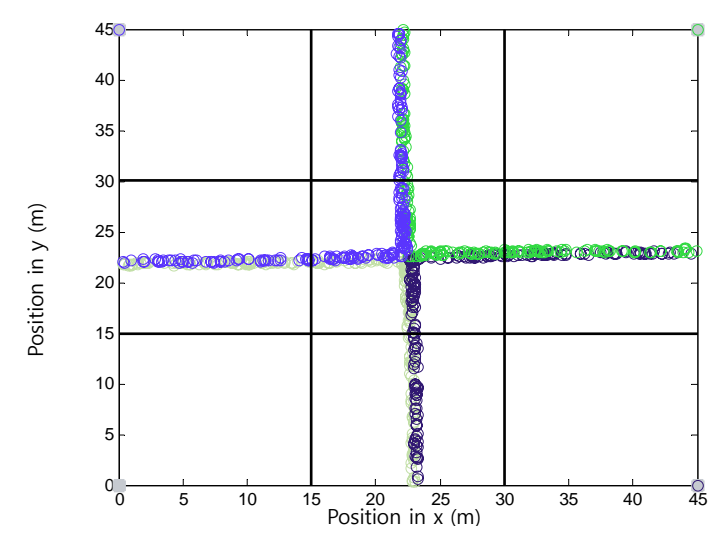

Fig. 4. Distribution of coordinated multi-point transmission and reception $($ CoMP) users when CoMP_ratio $=0.9$.

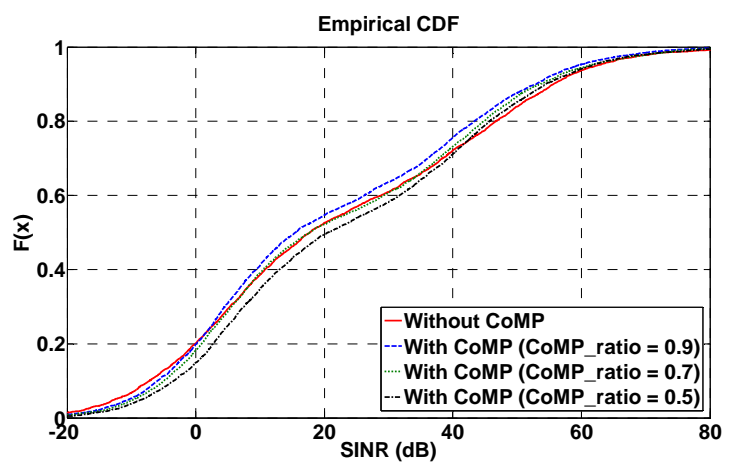

Fig. 5. Cumulative distribution function (CDF) of the effective signal-tointerference noise ratio (SINR) by varying CoMP_ratio.

Next, the cumulative distribution function (CDF) of the effective SINR has been shown in Fig. 5. The effective SINR is the SINR multiplied by the transmission opportunity. When CoMP is not used, the transmission opportunity does not deteriorate and the effective SINR is 
the same as the SINR itself. However, when the CoMP is used, the transmission opportunity will be lost and the effective SINR will be less than the SINR. For example, when CoMP_ratio is 0.9 , approximately $10 \%$ of users will use CoMP and the effective SINR is the SINR multiplied by 0.95. We used the notion of effective SINR to properly take into account the penalty in using the CoMP. Otherwise, we will obtain trivial results in which the SINR is always improved by using CoMP.

As can be seen from the results, the proportion of users that are in the low-SINR regime can be reduced by using the CoMP and the level of improvement is greater as more users use CoMP, that is, CoMP_ratio is small. However, at the same time, we also found that the ratio of users in the highSINR regime also decreases with CoMP because the transmission opportunities are lost by using CoMP and the level of deterioration is greater when more users use CoMP. As a result, the CDF becomes narrower if CoMP is used and the users can receive data more fairly.

Finally, we have evaluated the spectral efficiency of indoor mobile communication systems with CoMP by varying CoMP_ratio. We have evaluated the cell spectral efficiency and the user spectral efficiency for the users who belong to the lower $50 \%$ of spectral efficiency. The results are shown in Tables 2 and 3.

As we can see from the results, the average cell spectral efficiency is not improved by using CoMP, but rather it decreases. This is mainly due to the loss of the transmission opportunity in using the CoMP. However, as we can see from Table 3, the spectral efficiency of the lower $50 \%$ of users is improved by using CoMP (4.1\% for CoMP_ratio = 0.9 and $23.2 \%$ for CoMP_ratio $=0.7)$. The level of improvement is larger when more users use CoMP, that is, CoMP_ratio is small. Therefore, we can conclude that in indoor environments, CoMP can improve the performance

Table 2. Cell spectral efficiency

\begin{tabular}{lc}
\hline \multicolumn{1}{c}{ Cell spectral efficiency } & Value (bps/Hz/cell) \\
\hline Without CoMP & 2.6904 \\
CoMP $($ CoMP_ratio $=0.9)$ & 2.6196 \\
CoMP $($ CoMP_ratio $=0.7)$ & 2.5921 \\
\hline
\end{tabular}

CoMP: coordinated multi-point transmission and reception.

Table 3. Average spectral efficiency of the lower $50 \%$ of users

\begin{tabular}{lc}
\hline \multicolumn{1}{c}{ Cell spectral efficiency } & Value $(\mathbf{b p s} / \mathbf{H z} / \mathbf{u s e r})$ \\
\hline Without CoMP & 0.0858 \\
CoMP $($ CoMP_ratio $=0.9)$ & 0.0893 \\
CoMP $($ CoMP_ratio $=0.7)$ & 0.1057 \\
\hline
\end{tabular}

CoMP: coordinated multi-point transmission and reception. of low data rate users with the sacrifice of high data rate users and resources can be distributed more fairly. If the performance improvement of low data rate users is more important, then the CoMP_ratio can be set to a low value.

Although we did not include the results in this paper, we have also evaluated the performance of CoMP in outdoor cellular environments, and we found that the overall performance is also improved in that case. In indoor environments, the gain in spectral efficiency for low data rate users is not large enough to overcome the performance degradation caused by the loss of transmission opportunity in CoMP. The main reason for this phenomenon is that although the dominant interference can be efficiently removed by using CoMP, the amount of residual interference is still large. We can find from Fig. 3 that a huge portion of CoMP users is located in the room at the center of the building. In this region, the path loss of the signal from four BSs is likely to be similar. Therefore, even though the dominant interference is removed, the remaining interference is still huge and the performance gain of the CoMP diminishes. As a result, it should be noted that in indoor environments, more than two BSs should cooperate in transmission to achieve the full benefits of CoMP.

\section{DISCUSSION AND CONCLUSIONS}

In this paper, we have evaluated the performance of indoor mobile communication systems with CoMP technology. Although CoMP technology has been conceived as a key technology for future mobile communication systems, the performance of CoMP has not been evaluated in realistic indoor environments. For this purpose, we have designed a system-level simulator including specific realistic factors such as a practical antenna pattern and link-level results. Through intensive simulation, we have shown that unlike outdoor cellular systems, CoMP technology may improve the throughput of low data rate users; however, the overall throughput of mobile communication systems might deteriorate due to the loss of transmission opportunity.

\section{REFERENCES}

[1] "Cisco Visual Networking Index: global mobile data traffic forecast update, 2012-2017,” CISCO Systems Inc., San Jose, CA, White Paper, 2013.

[ 2 ] A. Goldsmith, Wireless Communications. New York, NY: Cambridge University Press, 2005.

[ 3 ] D. Tse and P. Viswanath, Fundamentals of Wireless Communications. New York, NY: Cambridge University Press, 2005. 
[ 4 ] S. Yun, Y. Yi, D. H. Cho, and J. Mo, "Open or close: on the sharing of femtocells," in Proceedings of IEEE INFOCOM, Shanghai, China, pp. 116-120, 2011.

[ 5 ] W. Lee and D. H. Cho, "Enhanced spectrum sensing scheme in cognitive radio systems with MIMO antennae," IEEE Transactions on Vehicular Technology, vol. 60, no. 3, pp. 1072-1085, 2011.

[6] W. Lee and D. H. Cho, “Adaptive interference estimation for directional transmission," in Proceedings of IEEE Consumer Communications and Networking Conference, Las Vegas, NV, pp. 350-351, 2012.

[ 7 ] R. Irmer, H. Droste, P. Marsch, M. Grieger, F. Fettweis, S. Brueck, H. P. Mayer, L. Thiele, and V. Jungnickel, “Coordinated multipoint: concepts, performance, and field trial results," IEEE Communications Magazine, vol. 49, no. 2, pp. 102-111, 2011.

[ 8 ] D. Lee, H. Seo, B. Clerckx, E. Hardouin, D. Mazzarese, S. Nagata, and K. Sayana, “Coordinated multipoint transmission and reception in LTE-advanced: deployment scenarios and operational challenges,” IEEE Communications Magazine, vol. 50, no. 2, pp. 148-155, 2012.

[ 9 ] B. Mondal, E. Visotsky, T. A. Thomas, X. Wang, and A. Ghosh, "Performance of downlink comp in LTE under practical constraints," in Proceedings of the IEEE 23rd International Personal
Indoor and Mobile Radio Communications, Sydney, Australia, pp. 2049-2054, 2012.

[10] Y. Gao, Y. Li, H. Yu, and S. Gao, "Performance of dynamic CoMP cell selection in 3GPP LTE system level simulation," in Proceedings of the IEEE 3rd International Conference on Communication Software and Networks, Xi'an, China, pp. 210-213, 2011.

[11] T. Kwon, H. Lee, S. Choi, J. Kim, D. H. Cho, S. Cho, S. Yun, W. H. Park, and K. H. Kim, "Design and implementation of a simulator based on a cross-layer protocol between MAC and PHY layers in a WiBro compatible IEEE 802.16e OFDMA system,” IEEE Communications Magazine, vol. 43, no. 12, pp. 136-146, 2005.

[12] "Guidelines for evaluation of radio interface technologies for IMTAdvanced,” ITU, Geneva, Switzerland, Report ITU-R M.2135, 2008.

[13] “3GPP TS 36.104 version 8.2.0. release 8: LTE; evolved Universal Terrestrial Radio Access (E-UTRA); base station (BS) radio transmission and reception,” European Telecommunications Standards Institute, Cedex, France, TS 136104 V8.2.0., 2008.

[14] M. Haenggi, “On distances in uniformly random networks,” IEEE Transactions on Information Theory, vol. 51, no. 10, pp. 35843586, 2005.

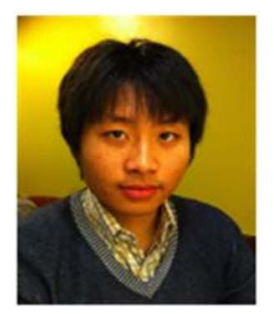

\section{Woongsup Lee}

received B.S. and Ph.D. degrees in electrical engineering from KAIST, Daejeon, Republic of Korea, in 2006 and 2012, respectively. He is currently with the Institute for Digital Communication at Friedrich Alexander University (FAU), Erlangen, Germany, as a postdoctoral researcher. His research interests include cognitive radio networks, future wireless communication systems, M2M networks, and smart grid systems.

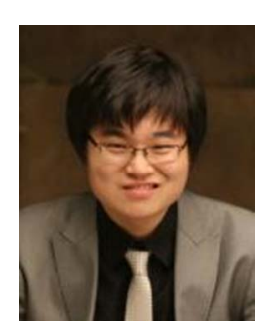

\section{Howon Lee}

is an assistant professor in the Department of Electrical, Electronic and Control Engineering at Hankyong National University. He received his B.S., M.S., and Ph.D. in electrical engineering from Korea Advanced Institute of Science and Technology (KAIST) in 2003, 2005, and 2009, respectively. Before joining HKNU, he was a team leader of the Knowledge Convergence Team at KAIST Institute for Information Technology (IT) Convergence until 2012. His current research interests include knowledge communications, IT convergence, cross-layer radio resource management, cognitive radio, and voice over IP (VoIP) service. He is also the recipient of the JCCl $2006 \mathrm{Best}$ Paper Award, Intel Student Paper Contest Bronze Prize in 2006, and TTA Paper Contest Encouragement Award in 2011. 